\title{
Age structure of annual Nothobranchius fishes in Mozambique: is there a hatching synchrony?
}

\author{
M. PolaČIK*†, M. T. Donner: AND M. ReICHARD* \\ *Institute of Vertebrate Biology, Academy of Sciences of the Czech Republic, Květná 8, \\ 60365 Brno, Czech Republic and \$Limnological Institute, University of Konstanz, 78464 \\ Konstanz, Germany
}

\begin{abstract}
The age structures of populations of African annual Nothobranchius spp. were examined for the first time. Daily increments in sagittal otoliths of Nothobranchius furzeri, Nothobranchius kadleci, Nothobranchius orthonotus and Nothobranchius rachovii from southern and central Mozambique were used for age determination. Four hypotheses were tested: (1) timing of hatching is consistent with the calendar onset of the rainy season, (2) hatching is synchronized within a population in a pool, (3) there is a difference in hatching date between geographical regions differing in mean total annual precipitation and (4) sympatric Nothobranchius spp. hatch at the same time. The results show that daily increment analysis represents an applicable method for age determination in Nothobranchius spp. Despite a significant positive relationship between age and size of fishes, a pronounced variation in fish size at an age precluded the use of fish size as a valid age marker. Timing of hatching was not consistent with the calendar onset of the rainy season. Interpopulation variability was observed in the degree of hatching date synchronization within a population. Hatching dates were relatively uniform in some populations, while there was considerable variability in others. Differences in timing of hatching date were found in only 1 of 2 years within the three regions investigated (Chefu, lower Limpopo and Sofala regions), each of which differed in mean total annual rainfall. The hatching dates of sympatric Nothobranchius spp. were marginally different, but further testing on a larger sample is needed for conclusive results.
\end{abstract}

Key words: age validation; ecology; killifishes; south-east Africa; temporary pools.

\section{INTRODUCTION}

The genus Nothobranchius (Cyprinodontiformes, Nothobranchiidae) represents a group of annual killifishes occurring in temporary savannah pools throughout east Africa. These small fishes survive the dry season as resistant eggs buried in the bottom substratum of desiccated pools. During the dry season, the eggs spend most of the time in a state of diapause, i.e. a state of dormancy with specific triggering and releasing conditions (Wourms, 1972; Watters, 2009; Podrabsky et al., 2010). Nothobranchius spp. are characterized by a system of three successive facultative diapauses

†Author to whom correspondence should be addressed. Tel.: +420 543422 522; email: polacik@ivb.cz 
that are sensitive to specific environmental cues (Wourms, 1972; Markofsky \& Matias, 1977; Levels et al., 1986; Watters, 2009). During the rainy season, the eggs hatch and fishes develop into reproductively active adults within as short a period as 3 weeks. Their life span is limited by the duration of the wet phase of the pool, which is typically several months (Wildekamp, 2004).

The genus Nothobranchius has great potential for experimental applications in developmental biology (Van Haarlem, 1979), toxicology (Shedd et al., 1999) and evolutionary biology (Haas, 1976; Reichard \& Polačik, 2010). As early as the 1970s, Nothobranchius guentheri (Pfeffer) was recognized as a promising model organism for the study of ageing processes due to its short maximum life span of c. 15 months (Markofsky \& Perlmutter, 1973). Only after the extremely short natural life span (only 9 weeks post-hatching) of Nothobranchius furzeri Jubb was discovered (Valdesalici \& Cellerino, 2003), however, did Nothobranchius spp. attract broader interest as a model group in ageing studies (Terzibasi et al., 2007; Hsu et al., 2008). Several laboratory studies have revealed marked differences in life span and expression of ageing biomarkers across different wild-derived $N$. furzeri strains, thus making this species a unique vertebrate for studying the genetic basis of these traits (Terzibasi et al., 2008; Valenzano et al., 2009).

Differences in life span and senescence rate across $N$. furzeri populations are considered to have a strong relationship with their respective geographical origins (Terzibasi et al., 2008). Within the distribution range of $N$. furzeri (Reichard et al., 2009), mean annual precipitation decreases primarily from east to west (Terzibasi et al., 2008). The habitats used by Nothobranchius spp. are considered to exist for a shorter period due to the lowered rainfall in the west and, consequently, this imposes selection pressure towards rapid maturation with consequent accelerated senescence and a shorter life span in western populations (Terzibasi et al., 2008). This hypothesis implies that the time of habitat existence should correlate strongly with life span of the fish. Support for this hypothesis is provided by the fact that captive, readyto-hatch Nothobranchius spp. embryos were always observed to hatch very quickly (minutes to hours) after contact with water (Podrabsky, 1999; M. Polačik, pers. obs.), implying that, in the wild, hatching should also occur immediately after a pool is filled with water.

Alternative opinions, however, suggest that the link between presence of water and occurrence of fishes may not be so straightforward. For example, Watters (2009) suggests that timing of Nothobranchius spp. hatching is not identical with the onset of the rainy season and that Nothobranchius spp. may hatch weeks after a pool is filled with water. There is a lack of supporting information, however, for either the former or the latter view, as no studies on the age structure of Nothobranchius spp. populations have been undertaken.

In this study, the age structures of four species of Nothobranchius: N. furzeri, Nothobranchius kadleci Reichard, Nothobranchius orthonotus (Peters) and Nothobranchius rachovii Ahl, from southern and central Mozambique (Fig. 1), are examined for the first time. Four hypotheses were tested: (1) timing of hatching is consistent with the calendar onset of the rainy season, (2) hatching is synchronized within a population in a pool, (3) there is a difference in hatching date between geographical regions differing in mean total annual precipitation and (4) sympatric Nothobranchius spp. hatch at the same time. 


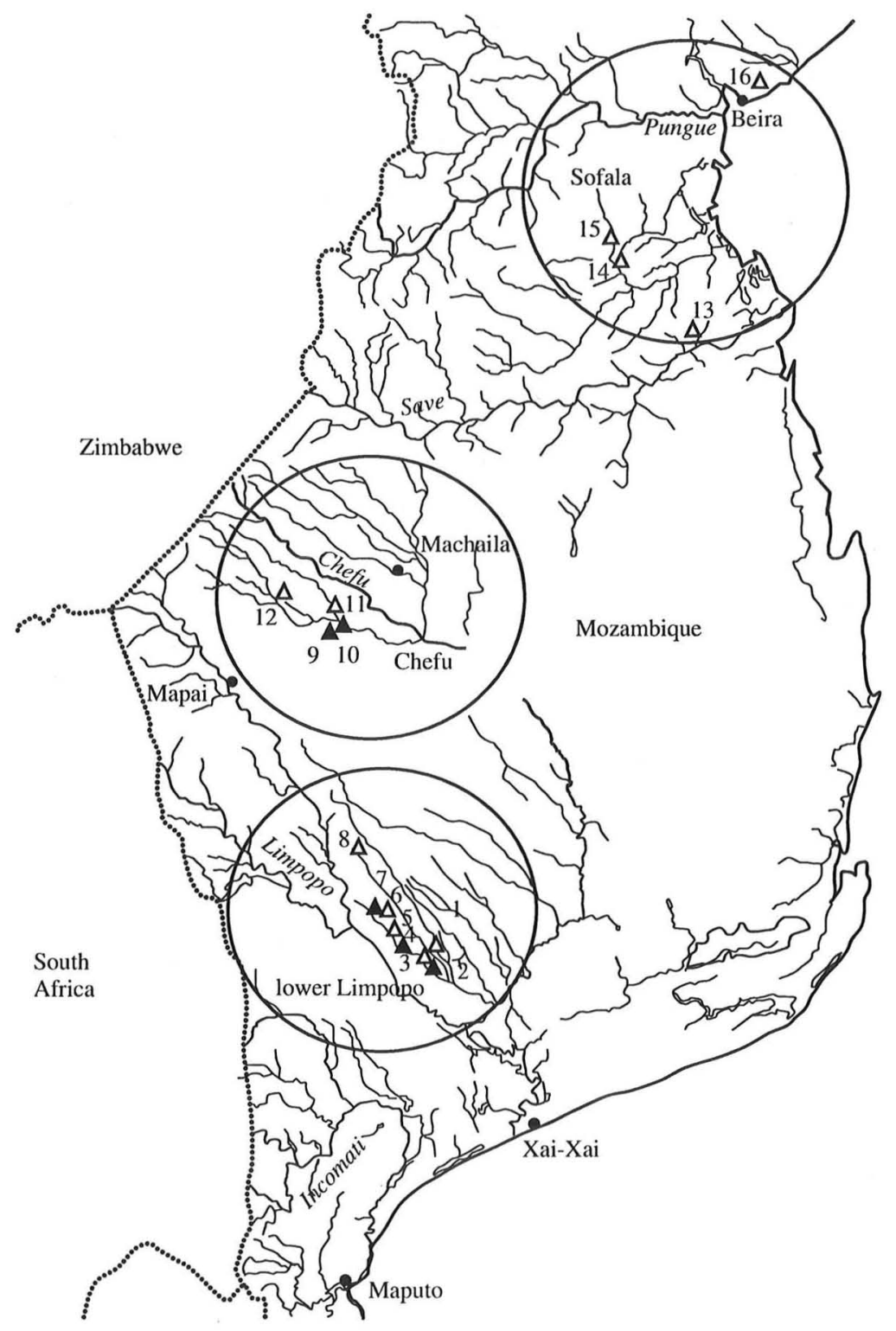

FIG. 1. Map of southern and central Mozambique, showing the river network and important settlements. National borders (......), and sites sampled in 2008 (five sites; $\mathbf{\Delta}$ ) and in 2009 (11 sites; $\Delta$ ) are indicated. The three regions within Nothobranchius spp. range that differ in mean annual rainfall are also indicated $(\mathrm{O})$. Note that many rivers are temporary and may not form flowing sections in every rainy season.

\section{MATERIALS AND METHODS}

The age structure of populations of Nothobranchius spp. was assessed through the determination of the number of daily increments in sagittal otoliths. A general validation of the relevance of daily increment readings in Nothobranchius spp. otoliths was performed using captive-reared fishes of known age. Both the sagittae and the lapilli of $N$. furzeri were checked 
for their suitability for daily increment readings, but lapilli showed only faint increments and consistent reading was impossible.

In order to confirm that the number of daily increments in an otolith reflects the real age of the fish in days, daily increments in the sagittae of $14 \mathrm{~N}$. furzeri specimens were read at seven successive ages ranging from 7 to 66 days (Fig. 2). In a pilot study, otoliths originating from specimens $>14$ days and raised under constant aquarium conditions were characterized by the presence of a large number of sub-daily increments. Two breeding approaches were employed to provide the fish with day-night temperature fluctuations [which may be important for otolith increment formation; Song et al. (2009)] as observed in the wild, where up to $15^{\circ} \mathrm{C}$ circadian fluctuations were recorded (Reichard et al., 2009).

First, fish up to an age of 13 days were maintained in a small aquarium and fed live Artemia sp. nauplii three times a day. On the 7 and 13 days, three individuals were sacrificed and their sagittae dissected and mounted on a microscope slide using epoxy resin. Second, in order to provide close to natural conditions for the fish, a group of $N$. furzeri was transferred to a 7001 tub filled with pond water. The tub was kept outside at the Institute of Vertebrate Biology in the Czech Republic during mid-summer to early autumn of 2009, thereby ensuring natural water temperature fluctuations (night temperature decreases) and the constant presence of natural food [aquatic invertebrates; Polačik \& Reichard (2010)], with only occasional food supplementation. The water temperature in the tub and its circadian fluctuations imitated natural day-night fluctuations (Reichard et al., 2009), with an overall amplitude between 12 and $31^{\circ} \mathrm{C}$. At selected time intervals (Fig. 2), sample individuals were removed and their sagittae mounted on microscope slides as for the aquarium-raised fish. All subsequent readings were undertaken blind, i.e. the slides were coded in order to prevent bias due to known age (captive fish) or origin (wild fish) of the fish.

The procedure for counting of daily increments involved careful polishing of the sagittae at low speed until the otolith core could be clearly identified. Subsequently, one of the sagittae was placed under a microscope and daily increments counted at a magnification of $\times 400$ to $\times 1250$. Counting was undertaken in a random manner and repeated twice, with at least a 1 month gap between the two readings. The mean value of the two readings was used for analyses.

Samples of wild Nothobranchius spp. were collected during two successive rainy seasons in 2008 and 2009 from southern and central Mozambique (Fig. 1), the natural distribution

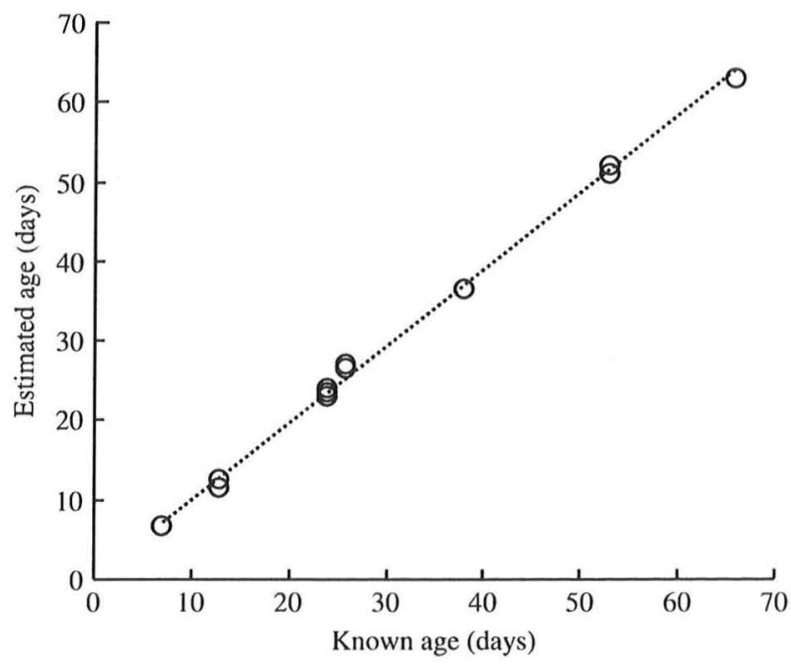

FIG. 2. Relationship between known age and age estimate, based on sagitta readings in captive Nothobranchius furzeri $(n=14)$. Note that a single point may represent more than one individual. The curve was fitted by $y=0.426+0.964 x$. 
TABLE I. Number $(n)$ and mean total length $\left(L_{\mathrm{T}}\right)$ of Nothobranchius spp., by year and site

\begin{tabular}{lrrrrrrrr}
\hline Species & Site & Year & $n_{\text {males }}$ & \multicolumn{1}{c}{$L_{\mathrm{T}}$} & S.D. & $n_{\text {females }}$ & $L_{\mathrm{T}}$ & S.D. \\
\hline N. furzeri & 1 & 2009 & 4 & $60 \cdot 3$ & $11 \cdot 9$ & 0 & & \\
& 2 & 2008 & 4 & $40 \cdot 0$ & $2 \cdot 7$ & 4 & $33 \cdot 0$ & $2 \cdot 5$ \\
& 3 & 2009 & 5 & $58 \cdot 4$ & $5 \cdot 4$ & 5 & $41 \cdot 8$ & $1 \cdot 2$ \\
& 4 & 2008 & 7 & $45 \cdot 0$ & $3 \cdot 9$ & 7 & $35 \cdot 0$ & $1 \cdot 1$ \\
& 5 & 2009 & 10 & \multicolumn{2}{c}{ No data } & 0 & & \\
& 6 & 2008 & 4 & $54 \cdot 5$ & $7 \cdot 2$ & 3 & $58 \cdot 8$ & $12 \cdot 0$ \\
& 7 & 2009 & 10 & $40 \cdot 8$ & $1 \cdot 9$ & 0 & & \\
& 8 & 2009 & 2 & $61 \cdot 0$ & $0 \cdot 0$ & 5 & $44 \cdot 2$ & $1 \cdot 6$ \\
& 9 & 2008 & 17 & $44 \cdot 6$ & $5 \cdot 4$ & 10 & $33 \cdot 8$ & $4 \cdot 8$ \\
& 10 & 2008 & 6 & $68 \cdot 2$ & $9 \cdot 0$ & 6 & $51 \cdot 8$ & $8 \cdot 0$ \\
& 11 & 2009 & 6 & $53 \cdot 5$ & $5 \cdot 7$ & 4 & $41 \cdot 5$ & $1 \cdot 5$ \\
Nadleci & 12 & 2009 & 10 & $40 \cdot 5$ & $3 \cdot 0$ & 0 & & \\
& 13 & 2009 & 5 & $54 \cdot 4$ & $3 \cdot 3$ & 5 & $43 \cdot 0$ & $1 \cdot 1$ \\
& 14 & 2009 & 3 & $67 \cdot 0$ & $2 \cdot 4$ & 5 & $55 \cdot 2$ & $2 \cdot 4$ \\
& 15 & 2009 & 10 & $46 \cdot 5$ & $1 \cdot 4$ & 0 & & \\
& 1 & 2009 & 2 & $72 \cdot 5$ & $2 \cdot 5$ & 8 & $70 \cdot 9$ & $6 \cdot 1$ \\
& 5 & 2009 & 4 & $37 \cdot 8$ & $1 \cdot 3$ & 0 & & \\
N. rachovii & 6 & 2008 & 3 & $62 \cdot 7$ & $12 \cdot 7$ & 7 & $59 \cdot 5$ & $10 \cdot 3$ \\
& 16 & 2009 & 5 & $41 \cdot 4$ & $2 \cdot 4$ & 5 & $39 \cdot 8$ & $1 \cdot 5$ \\
& 5 & 2009 & 2 & $29 \cdot 5$ & $2 \cdot 5$ & 0 & & \\
& 12 & 2009 & 10 & $33 \cdot 6$ & $2 \cdot 1$ & 0 & & \\
& 16 & 2009 & 4 & $33 \cdot 3$ & $0 \cdot 8$ & 4 & $27 \cdot 5$ & $1 \cdot 1$ \\
\hline
\end{tabular}

range of the four species (Reichard et al., 2009). The rainy season in Mozambique starts in October and ends in April (Watters, 2009); therefore, sampling took place between 8 and 22 February 2008 and between 6 and 26 February 2009. Five sites and two Nothobranchius spp. were sampled in 2008, and 11 sites and four Nothobranchius spp. in 2009, using both seine and dip-nets (Table I).

The sites sampled were situated in three regions differing in mean annual rainfall, as this was hypothesized as having an influence on the hatching date of the sampled Nothobranchius spp. populations. The semi-arid Chefu region, situated further from the shore of the Indian Ocean and at a higher altitude, receives less than half $(c .400 \mathrm{~mm})$ the annual mean rainfall of the lower Limpopo floodplain, where annual rainfall may locally exceed $1000 \mathrm{~mm}$ (Watters, 2009). The Sofala region, the most humid of the sites with annual rainfall from 800 to $1200 \mathrm{~mm}$ (Anon., 2007), is located in central Mozambique.

After capture, a randomly chosen sub-sample of fishes $(n=2-27)$ were anaesthetized with clove oil, measured (total length, $L_{\mathrm{T}}$ ) and preserved in $70 \%$ ethanol. In the laboratory, the otoliths from the preserved fishes were treated in the same way as those used for validation, with the exception of samples collected in 2008 where thermoactive Crystalbond (Aremco Products, Inc.; www.aremco.com) was used for otolith mounting instead of epoxy resin.

\section{RESULTS}

Age determination based on otoliths from $N$. furzeri raised under laboratory conditions was precise over the entire range of ages investigated (7-66 days). The calibration showed a near-perfect correlation between known age and age estimated 
from daily otolith increments $(r=0.99, n=14$; Fig. 2). The maximum difference between two readings was 6 days (mean \pm s.D. $=1 \cdot 3 \pm 1 \cdot 5$ ).

The appearance and readability of otoliths originating from wild fishes was variable in terms of shape, colour and clarity of increment structure. Some wild fish otoliths were difficult or even impossible to read, and this was consistent across individual fishes at particular sites, e.g. N. orthonotus at sites 5 and 10 and N. furzeri at sites 2, 4 and 5 (Table II). On the other hand, otoliths from some sites were easily readable even between different Nothobranchius spp., e.g. at sites 11 and 16.

No difference was observed between birth date estimates for male and female $N$. furzeri $(t$-test, d.f. $=99, P>0 \cdot 05)$. Despite this, males were larger than females $(t$-test, d.f. $=90, P<0.05)$, attaining a mean \pm s.E. of $48.8 \pm 1.3 \mathrm{~mm}$ compared to $42.9 \pm 1.8 \mathrm{~mm}$ for females. Nothobranchius furzeri $L_{\mathrm{T}}$ was positively correlated with age estimates in males and females (Fig. 3), but the proportion of variability accounted for by the statistical model was low (linear regression: $r^{2}=0 \cdot 126, P<$ 0.01 and $r^{2}=0.301, P<0.01$ for males and females, respectively).

The mean birth date did not vary between years for $N$. furzeri (ANOVA, d.f. = $1,102, P>0.05$ ), with a mean birth date of 31 December 2007 ( \pm 1 day, $n=47$, range: 14 December 2007 to 15 January 2008) for the 2008 sampling and 1 January 2009 ( \pm 1 day, $n=59$, range: 19 December 2008 to 19 January 2009) for the 2009 sampling. The age of $N$. furzeri from individual pools at some sampling sites varied by up to 25 days between the youngest and oldest fish, though it was more consistent in other pools, with a maximum difference of 5 to 9 days between the youngest and the oldest fish (Table II). All captured fish in the pools sampled were sexually mature.

In 2008 , the mean birth date estimates for $N$. furzeri did not differ across populations [ANOVA, d.f. $=4,42, P>0.05$; Fig. 4(a)]. In 2009, however, mean estimates of fish birth date varied significantly across populations (ANOVA, d.f. $=5,48$, $P<0.001$ ), with $N$. furzeri from the Chefu region (site 12) being $c .1$ week younger than $N$. furzeri from the Limpopo region (sites 3 and 5). The inclusion of $N$. kadleci (a northern, sister species of $N$. furzeri from the wet region) into the analysis did not change the overall interpretation (ANOVA, d.f. $=8,68, P<0.001$ ), $N$. kadleci fish falling into the range of $N$. furzeri birth date estimates [Fig. 4(b)].

At two sites, a comparison of birth dates was possible between two sympatric species. At site 11, N. rachovii was estimated to hatch 3 days earlier than $N$. furzeri. While this difference was statistically significant [ANOVA, d.f. $=1,18, P<0.05$; Fig. 5(a)], a large overlap in estimated birth dates between the two species was also characteristic. At site $16, N$. orthonotus was estimated to hatch 6 days earlier than $N$. rachovii [ANOVA, d.f. $=1,16, P<0.001$; Fig. 5(b)]. At three other sites where $N$. rachovii and $N$. orthonotus ages were estimated (sites 5, 6 and 10), low sample sizes precluded statistical testing with $N$. furzeri, but all fishes fell within the range of age estimates for $N$. furzeri (Table II).

\section{DISCUSSION}

The results indicate that the use of daily increments in sagittal otoliths represents an applicable method of age determination in Nothobranchius spp. Although there was a significant linear relationship between the age and size of the fishes, marked variation in fish size at respective ages precluded the use of fish size as a valid age 
TABLE II. Age structure of Nothobranchius spp. populations by year and geographic region

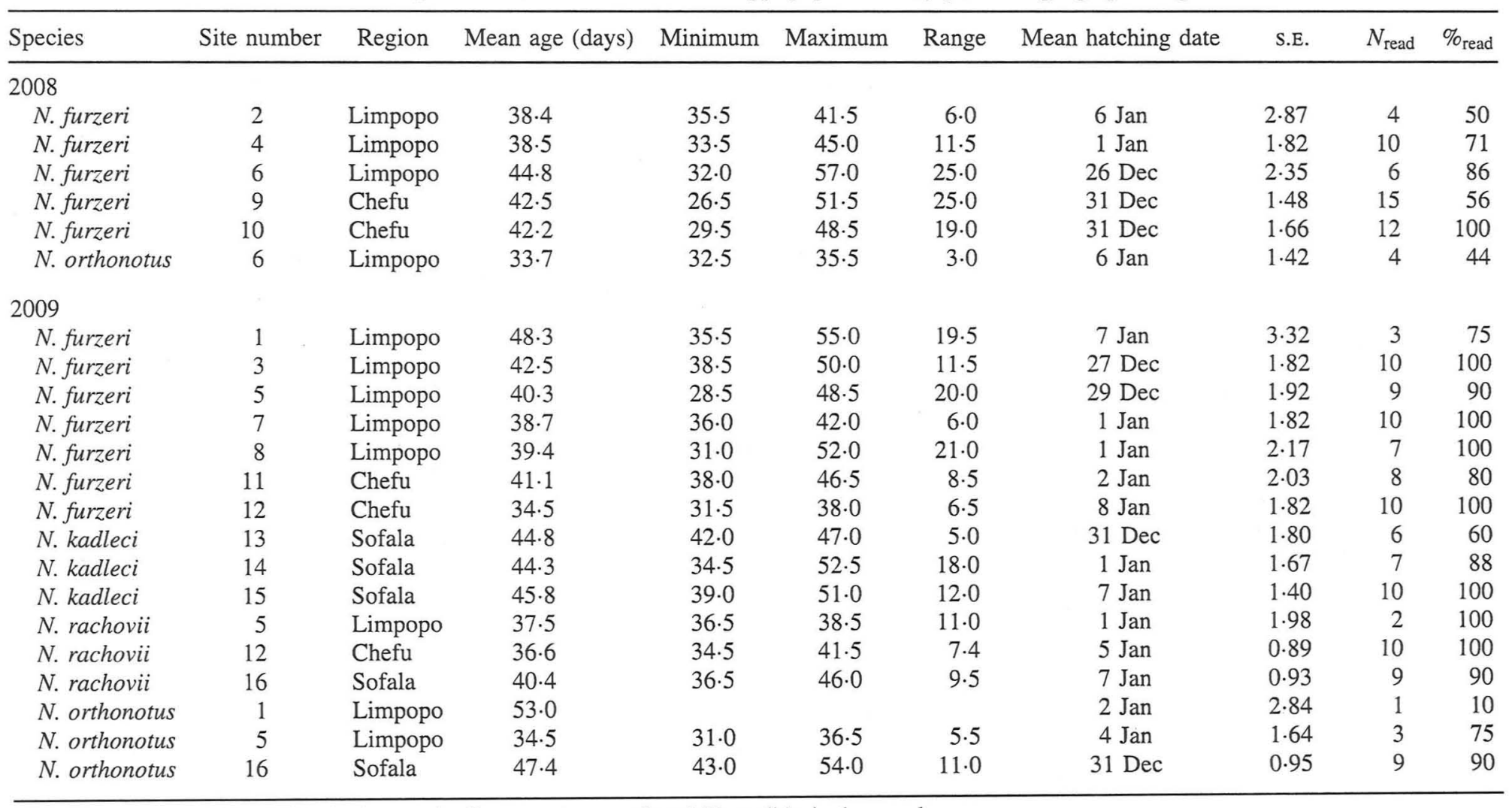

$N_{\text {read }}$, number of readable otoliths in the sample; $\%_{\text {read }}$, percentage of readable otoliths in the sample. 


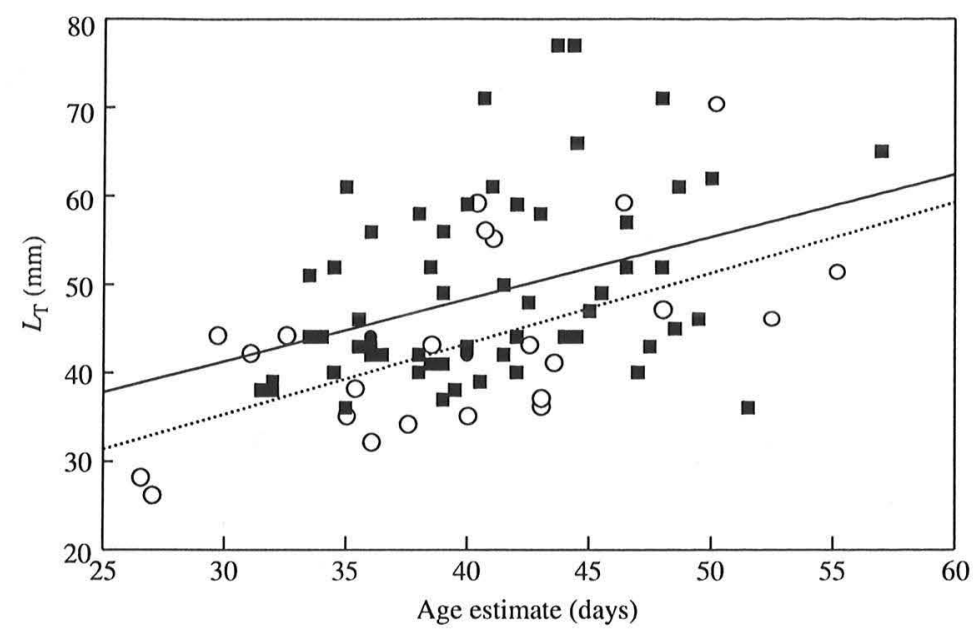

FIG. 3. Relationship between total length $\left(L_{T}\right)$ and age in Nothobranchius furzeri males (घ) and females $(O)$. The curves were fitted by: males (—) $y=20 \cdot 16+0.71 x$ and females (.....) $y=11.42+0.80 x$.

marker. The timing of hatching was not consistent with the calendar onset of the rainy season. In $N$. furzeri, there was interpopulation variability in the degree of hatching date synchronization within a population. In some populations, the hatching dates were relatively uniform while there was considerable variability in others. Different timing of the hatching date was found between those regions differing in mean annual rainfall in only one of the two seasons examined. The hatching dates of sympatric Nothobranchius spp. were marginally different, but further testing on a larger sample size is needed.

With regard to the data obtained from captive $N$. furzeri (Fig. 2), the accuracy of age determination based on daily increments in sagittae was very good within the range tested (7-66 days). Accuracy depended mainly on the readability of the otolith, which reached a relatively high level in the validation sample. On the other hand, as indicated by both the pilot study (see above) and the highly variable appearance and readability of otoliths from wild fishes, good otolith readability does not seem to be a typical species characteristic of the Nothobranchius spp. examined, unlike other fish species such as Perca fluviatilis L. (Kristensen et al., 2008) or Sprattus sprattus (L.) (Baumann et al., 2006). Environmental conditions often vary considerably between populations of Nothobranchius spp. (Reichard et al., 2009; Watters, 2009; Reichard, 2010), and this variability probably plays an important role in determining the otolith characteristics in Nothobranchius spp., as is the case in many other fish species (Klink \& Eckmann, 1992; Graynoth, 1999; Gonzalez-Salas \& Lenfant, 2007).

The use of body size as an age marker does not appear to be appropriate in Nothobranchius spp. Although there was a statistically significant relationship between $L_{\mathrm{T}}$ of the fish and its age in $N$. furzeri, it explained only a small proportion of the variation observed (Fig. 3). A plastic growth rate in relation to age was expected as growth in these fishes, which have a very fast metabolism, is known to be sensitive to food availability and quality, and density of conspecifics (Inglima et al., 1981; 


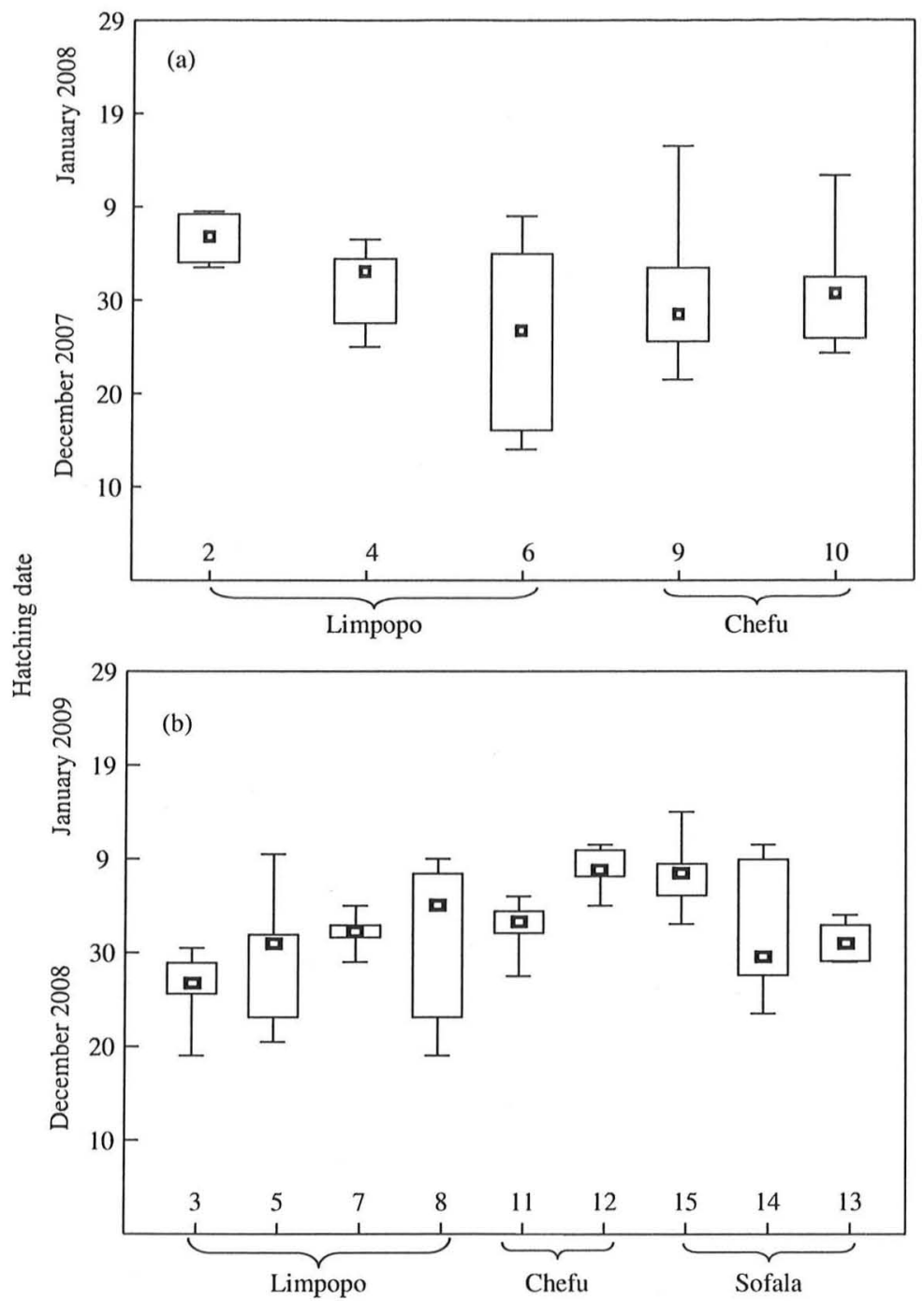

FIG. 4. Mean hatching dates, with median (centre point), interquartile range (box) and total range (whiskers), of Nothobranchius furzeri populations by year of sampling: (a) 2008 and (b) 2009, and region.

Genade et al., 2005). The coefficient of determination showed that the proportion of variability in the data explained by the regression model was more than two times higher in females than in males. This is most likely due to the existence of a hierarchical structure among males (Polačik \& Reichard, 2009), resulting in differential growth trajectories for males and females (Hamilton \& Heg, 2008; Graf et al., 2010). Growth rates of Nothobranchius spp. in the wild are similar to those reported from captivity, as all fishes in the pools sampled (minimum determined age 26.5 days) were sexually mature. In captivity, some $N$. furzeri strains reach sexual maturity within 3 to 4 weeks post-hatching (Valdesalici \& Cellerino, 2003). The data on the age of wild $N$. furzeri presented in this study (all individuals sexually mature) 


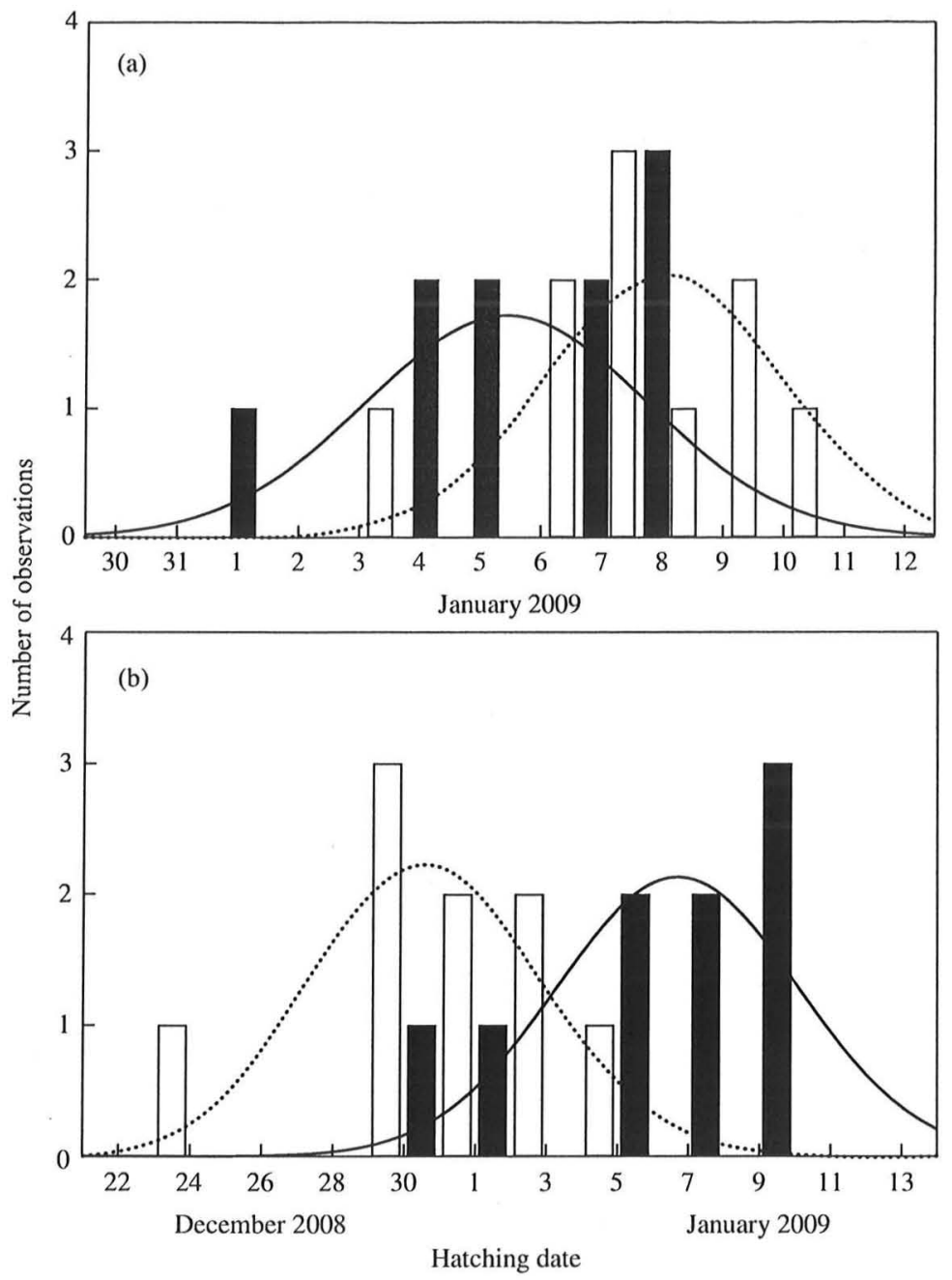

FIG. 5. Age structure of sympatric Nothobranchius spp. populations, represented by the frequency distribution of hatching dates of (a) N. furzeri ( $\square$ ) and N. rachovii ( $\square$ ) at site 12 and (b) N. orthonotus ( $\square$ ) and

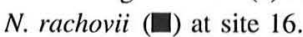

support the view that this vertebrate has the world's earliest attainment of sexual maturity (Kon \& Yoshino, 2002; Depczynski \& Bellwood, 2005).

The data presented support the view that Nothobranchius spp. do not hatch immediately after the first rains at the very start of the rainy season. The mean hatching dates of all fishes were from mid-December to mid-January in both years sampled, i.e. the peak period in rainfall in Mozambique rather than the beginning of the rainy season, which occurs as early as October and ends in April (Watters, 2009). Hatching in the peak period of the rainy season is likely to represent a reasonable trade-off between the probability of habitat desiccation after the initial rains and the probability that the habitat will last long enough for fishes to mature and reproduce. 
Some variation in hatching date in fishes originating from the same pool was found in all four Nothobranchius spp. examined, indicating that hatching is not entirely synchronized within a population. On the other hand, the order of magnitude of asynchrony was not substantial and all fishes within a pool were sexually mature. There are two possible mechanisms (which are not mutually exclusive and may act in combination) to explain how a single Nothobranchius spp. population may show asynchrony in hatching date. The first assumes that Nothobranchius spp. eggs are already fully developed at the onset of the rainy season and that they hatch soon after they come into contact with water. Under these conditions, hatching date asynchrony would only be ascribed to a progressive inundation of a pool, with eggs laid in the deepest part hatching first, or as a result of the merging of several adjacent pools accumulating water at different rates (Watters, 2009). The main assumption of the second mechanism is that, at the onset of the rainy season, the majority of Nothobranchius spp. eggs are not yet ready to hatch, but are still resting in a dormant stage, i.e. in diapause. Moisture from the first rains provides the stimulus for the continuation of embryo development and escape from the diapause stage (Watters, 2009). In this case, hatching date asynchrony would arise because Nothobranchius spp. eggs are at various stages of development during the first rains. This is plausible as Nothobranchius spp. embryos may proceed through three facultative diapauses during egg incubation (Wourms, 1972) and egg developmental time, at least in captivity, varies within a single clutch (Wildekamp, 2004).

In concordance with the general tendency of the Nothobranchius spp. examined to hatch at the peak of the rainy season, the mean hatching date appears relatively stable across those regions with different mean annual rainfall totals. The range of $N$. furzeri (Reichard et al., 2009) allowed for a test as to whether there was any difference in the mean hatching date between two areas differing in annual rainfall totals. Despite the contrast in rainfall, there was no difference in the mean hatching date between the drier Chefu and the more humid Limpopo region (see above) in 2008 and only a marginal difference of 1 week in 2009 (Fig. 4). This is in contrast with the large differences in age-associated changes in behaviour and histology between captive strains derived from populations from the two regions (Terzibasi et al., 2008). Moreover, although all $N$. kadleci and some $N$. orthonotus and $N$. rachovii populations were found in the most humid of the sampled regions, the Sofala region, their mean hatching date fell within the range of $N$. furzeri hatching dates (Table II). The interregional congruence in mean hatching date indicates that, if the differences in life span and ageing rate of Nothobranchius spp. populations are affected by habitat duration (Terzibasi et al., 2008), the time of pool desiccation (earlier in drier regions) rather than hatching date (or beginning of the rainy season) represents the important selective pressure. Alternatively, the ageing and life span differences among populations may have evolved during a relatively drier glacial period, when the difference between the coastal and inland areas was more pronounced, implying stronger selective pressure compared to the present (Finch \& Hill, 2008). Currently, the selection pressure on life span via habitat duration may be relaxed.

Life-history theory (Roff, 2002) predicts that shorter habitat persistence in regions that receive less rain could lead to selection for earlier hatching and a higher level of hatching synchrony as late hatching fishes should be disadvantaged by a higher risk of desiccation. It should be noted, however, that dry regions within the Nothobranchius spp. range are also characterized by lower predictability of rainfall 
(Wildekamp, 2004; Mazuze, 2007; Watters, 2009) and the erratic seasonal pattern of rains is expected to select for lower hatching synchrony. On both the interspecific and intraspecific levels therefore, Nothobranchius spp. from drier regions are likely to respond to environmental unpredictability by developmental desynchronization (Watters, 2009). Hence, the selection for early hatching in dry region populations may have been restrained by selection for developmental asynchrony.

Interspecific differences in hatching date between sympatric $N$. furzeri and $N$. rachovii, and between $N$. rachovii and $N$. orthonotus, were statistically significant, but minor, at 3 and 6 days, respectively. With regard to the fast growth rate of Nothobranchius spp., however, even a difference of a few days could be biologically important as it may allow for potential predation of the earlier hatching species on its congeners. In particular, this could be the case for $N$. orthonotus, a large predatory Nothobranchius spp. that, as an adult, feeds also on tadpoles and lungfish Protopterus annectens (Owen) in the wild (Polačik \& Reichard, 2010) and in captivity is capable of cannibalism among juveniles (M. Polačik, pers. obs.). An alternative explanation is that the shift in mean hatching date simply reflects interspecific differences in the rate of development once the escape from a diapause has been triggered (see above). These hypotheses need to be tested in the field.

The environmental conditions experienced by Nothobranchius spp., and their adaptations to them, have made these fishes a valuable laboratory model in recent years. The increasing employment of Nothobranchius spp. as models for pharmacological, genomic and evolutionary research in the laboratory highlights, however, the importance of studies on the ecology in the wild. Only with a robust knowledge of Nothobranchius spp. ecology and demography in the wild can laboratory data be soundly interpreted and further insights into the biology of these species be gained.

This study was funded by project 206/09/0815 of the Grant Agency of the Czech Republic. The authors thank R. Eckmann's laboratory at the Limnological Institute, University of Konstanz, Germany, for providing facilities for work, and especially M. Schmid for her great help with sample processing and J. Frouzová for an introduction to otolith processing.

\section{References}

Baumann, H., Hinrichsen, H. H., Voss, R., Stepputtis, D., Grygiel, W., Clausen, L. W. \& Temming, A. (2006). Linking growth to environmental histories in central Baltic youngof-the-year sprat, Sprattus sprattus: an approach based on otolith microstructure analysis and hydrodynamic modelling. Fisheries Oceanography 15, 465-476. doi: 10.1111/ j.1365-2419.2005.00395.x

Depczynski, M. \& Bellwood, D. R. (2005). Shortest recorded vertebrate lifespan found in a coral reef fish. Current Biology 15, 288-289.

Finch, J. M. \& Hill, T. R. (2008). A late quaternary pollen sequence from Mfabeni Peatland, South Africa: reconstructing forest history in Maputaland. Quaternary Research 70, 442-450.

Genade, T., Benedetti, M., Terzibasi, E., Roncaglia, P., Valenzano, D. R., Cattaneo, A. \& Cellerino, A. (2005). Annual fishes of the genus Nothobranchius as a model system for aging research. Aging Cell 4, 223-233. doi: 10.1111/j.1474-9726.2005.00165.x

Gonzalez-Salas, C. \& Lenfant, P. (2007). Interannual variability and interannual stability of the otolith shape in European anchovy Engraulis encrasicolus (L.) in the Bay of Biscay. Journal of Fish Biology 70, 35-49. doi: 10.1111/j.1095-8649.2006.01243.x

Graf, M., Cellerino, A. \& Englert, C. (2010). Gender separation increases somatic growth in females but does not affect lifespan in Nothobranchius furzeri. PLoS ONE 5, e11958. doi: 10.1371/journal.pone.0011958 
Graynoth, E. (1999). Improved otolith preparation, ageing and back-calculation techniques for New Zealand freshwater eels. Fisheries Research 42, 137-146.

van Haarlem, R. (1979). Contact inhibition of overlapping: one of the factors involved in deep cell epiboly of Nothobranchius korthausae. Developmental Biology 70, 171-179.

Haas, R. (1976). Sexual selection in Nothobranchius guentheri (Pisces-Cyprinodontidae). Evolution 30, 614-622.

Hamilton, I. M. \& Heg, D. (2008). Sex differences in the effect of social status on the growth of subordinates in a co-operatively breeding cichlid. Journal of Fish Biology 72, 1079-1088. doi: 10.1111/j.1095-8649.2007.01787.x

Hsu, C. Y., Chiu, Y. C., Hsu, W. L. \& Chan, Y. P. (2008). Age-related markers assayed at different developmental stages of the annual fish Nothobranchius rachovii. Journals of Gerontology 63, 1267-1276.

Inglima, K., Perlmutter, A. \& Markofsky, J. (1981). Reversible stage-specific embryonic inhibition mediated by the presence of adults in the annual fish Nothobranchius guentheri. Journal of Experimental Zoology 215, 23-33.

Klink, A. \& Eckmann, R. (1992). Limits for the detection of daily otolith increments in whitefish (Coregonus lavaretus L.) larvae. Hydrobiologia 231, 99-105.

Kon, T. \& Yoshino, T. (2002). Extremely early maturity found in Okinawan gobioid fishes. Ichthyological Research 49, 224-228.

Kristensen, P. B., Closs, G. P., Lokman, P. M. \& Gronkjaer, P. (2008). Otolith formation, microstructure and daily increment validation in juvenile perch Perca fluviatilis. Journal of Fish Biology 73, 1478-1483. doi: 10.1111/j.1095-8649.2008.02019.x

Levels, P. J., Gubbels, R. E. \& Denuce, J. M. (1986). Oxygen consumption during embryonic development of the annual fish Nothobranchius korthausae with special reference to diapause. Comparative Physiology and Biochemistry 84, 767-770.

Markofsky, J. \& Matias, J. R. (1977). The effects of temperature and season of collection on the onset and duration of diapause in embryos of the annual fish Nothobranchius guentheri. Journal of Experimental Zoology 202, 49-56.

Markofsky, J. \& Perlmutter, A. (1973). Growth differences in subgroups of varying longevities in a laboratory population of the male annual cyprinodont fish, Nothobranchius guentheri (Peters). Experimental Gerontology 8, 65-73.

Podrabsky, J. E. (1999). Husbandry of the annual killifish Austrofundulus limnaeus with special emphasis on the collection and rearing of embryos. Environmental Biology of Fishes 54, 421-431.

Podrabsky, J. E., Garrett, I. D. F. \& Kohl, Z. F. (2010). Alternative developmental pathways associated with diapause regulated by temperature and maternal influences in embryos of the annual killifish Austrofundulus limnaeus. Journal of Experimental Biology 213, 3280-3288. doi: $10.1242 /$ jeb.045906

Polačik, M. \& Reichard, M. (2009). Indirect fitness benefits are not related to male dominance in a killifish. Behavioral Ecology and Sociobiology 63, 1427-1435. doi: 10.1007/ s00265-009-0798-2

Polačik, M. \& Reichard, M. (2010). Diet overlap among three sympatric African annual killifish species (Nothobranchius spp.) from Mozambique. Journal of Fish Biology 77, 754-768. doi: 10.1111/j.1095-8649.2010.02717.x

Reichard, M. (2010). Nothobranchius kadleci (Cyprinodontiformes: Nothobranchiidae), a new species of annual killifish from central Mozambique. Zootaxa 2332, 49-60.

Reichard, M. \& Polačik, M. (2010). Reproductive isolating barriers between colourdifferentiated populations of an African annual killifish, Nothobranchius korthausae (Cyprinodontiformes). Biological Journal of the Linnean Society 100, 62-72.

Reichard, M., Polačik, M. \& Sedláček, O. (2009). Distribution, colour polymorphism and habitat use of the African killifish Nothobranchius furzeri, the vertebrate with the shortest life span. Journal of Fish Biology 74, 198-212. doi: 10.1111/j.1095-8649.2008. 02129.x

Roff, D. A. (2002). Life History Evolution. Sunderland, MA: Sinauer Associates.

Shedd, T. R., Widder, M. W., Toussaint, M. W., Sunkel, M. C. \& Hull, E. (1999). Evaluation of the annual killifish Nothobranchius guentheri as a tool for rapid acute toxicity screening. Environmental Toxicology and Chemistry 18, 2258-2261. 
Song, Z., Fu, Z., He, C., Shen, D. \& Yue, B. (2009). Effects of temperature, starvation and photoperiod on otolith increments in larval Chinese sucker, Myxocyprinus asiaticus. Environmental Biology of Fishes 84, 159-171. doi: 10.1007/s10641-008-9403-1

Terzibasi, E., Valenzano, D. R. \& Cellerino, A. (2007). The short-lived fish Nothobranchius furzeri as a new model system for aging studies. Experimental Gerontology 42, 81-89. doi: 10.1016/j.exger.2006.06.039

Terzibasi, E., Valenzano, D. R., Benedetti, M., Roncaglia, P., Cattaneo, A., Domenici, L. \& Cellerino, A. (2008). Large differences in aging phenotype between strains of the short-lived annual fish Nothobranchius furzeri. PLOS ONE 3, 1-12. doi: 10.1371/journal.pone. 0003866

Valenzano, D. R., Kirschner, J., Kamber, R. A., Zhang, E., Weber, D., Cellerino, A., Englert, C., Platzer, M., Reichwald, K. \& Brunet, A. (2009). Mapping loci associated with tail color and sex determination in the short-lived fish Nothobranchius furzeri. Genetics 183, 1385-1395. doi: 10.1534/genetics.109.108670

Valdesalici, S. \& Cellerino, A. (2003). Extremely short lifespan in the annual fish Nothobranchius furzeri. Proceedings of the Royal Society B 270, 189-191. doi: 10.1098/rsbl. 2003.0048

Watters, B. R. (2009). The ecology and distribution of Nothobranchius fishes. Journal of the American Killifish Association 42, 37-76.

Wildekamp, R. H. (2004). A World of Killies: Atlas of the Oviparous Cyprinodontiform Fishes of the World, Vol. 4. Elyria, OH: American Killifish Association.

Wourms, J. (1972). The developmental biology of annual fishes. III. Pre-embryonic and embryonic diapause of variable duration in the eggs of annual fishes. Journal of Experimental Zoology 182, 389-414.

\section{Electronic References}

Anon. (2007). Estratégia ambiental para o desenvolvimento sustanável de Moçambique. Meputo: Ministério para a coordenação da acço ambiental. Available at http://www. legisambiente.gov.mz/index.php?option=com_docman\&task=doc_view\&grid=137/ (accessed 24 November 2010).

Mazuze, F. M. (2007). Analysis of Adoption of Orange-fleshed Sweetpotatoes: The Case Study of Gaza Province in Mozambique. Available at http://www.aec.msu.edu/fs2/ mozambique/MAZUZE_MS.pdf/ (accessed 24 November 2010). 